\title{
Calidad de forraje de canola (Brassica napus L.) en floraciones temprana y tardía bajo condiciones de temporal en Zacatecas, México
}

\section{Forage quality of canola (Brassica napus L.) at early and late bloom under rainfed conditions in Zacatecas, Mexico}

\author{
Alejandro Espinoza-Canales ${ }^{a}$, Héctor Gutiérrez-Bañuelos ${ }^{a *}$, Ricardo A. Sánchez-Gutiérrez ${ }^{b}$, \\ Alberto Muro-Reyes ${ }^{a}$, Francisco J. Gutiérrez-Piñaa ${ }^{a}$ Agustín Corral-Lunac
}

\begin{abstract}
RESUMEN
El objetivo fue evaluar la composición nutricional y el potencial forrajero de la canola en condiciones de temporal, durante dos etapas de crecimiento. Se utilizaron 48 muestras seleccionadas al azar de forraje en floración temprana ( $5 \%$ floración, $n=24)$ y tardía (90\% floración, $n=24)$, recolectadas de una parcela ( 1 ha) situada en el municipio de Calera de Víctor Rosales, Zacatecas, México, caracterizado por ser un clima semi-templado. Todas las muestras se secaron a $65{ }^{\circ} \mathrm{C}$ durante $48 \mathrm{~h}$; las evaluaciones nutricionales y bromatológicas se realizaron utilizando métodos estándar. Se observaron diferencias $(P<0.05)$ en todas las variables estudiadas; la proteína cruda $(\%)$ promedió 26 vs 12, fibra detergente neutro (\% base seca) 47 vs 60, fibra detergente ácido (\% base seca) 28 vs 52 , consumo esperado (DMI, \% PV) 2.56 vs 1.99, digestibilidad de la materia seca (\% base seca) 67 vs 48, valor relativo del forraje 133 vs 72, y la energía neta de mantenimiento (Mcal/ $\mathrm{kg}$ materia seca) 1.52 vs 0.86 , respectivamente para la floración temprana y tardía. Se concluye que el forraje de la canola en la fase de floración temprana presenta una mayor calidad como resultado de sus contenidos de proteína cruda y de la fracción fibra. Comparado con la floración tardía, el valor energético estimado resultó 1.8 veces mayor (1.52 vs $0.86 \mathrm{Mcal} \mathrm{ENm} / \mathrm{kg}$ ) para la floración temprana, este valor energético estimado es similar al del pasto sudán.
\end{abstract}

PALABRAS CLAVE: Canola, Potencial forrajero, Perfil nutricional, Proteína, Energía.

\begin{abstract}
Canola forage could be an option for diversify crops grown under rainfed conditions and to improve quality forage produced. The objective was to evaluate nutritional composition of canola under rainfed conditions at two plantgrowing stages. Fourty eight (48) randomly samples of forage in early bloom ( $5 \%$ bloom, $n=24)$ and late bloom ( $90 \%$ bloom, $n=24$ ) were used within 1 ha plot located in semi-temperate climate at North of Mexico. All samples were dry at $65{ }^{\circ} \mathrm{C}$ during $48 \mathrm{~h}$; bromatological and nutritional evaluation were performed using standard methods. Differences $(P<0.05)$ were observed in all variables, averaging 26 vs $12 \%$ crude protein, 47 vs $60 \%$ neutral detergent fiber, 28 vs $52 \%$ acid detergent fiber, 2.56 vs $1.99 \%$ dry matter intake (\%BW), 67 vs $48 \%$ DMS, and 133 vs 72 relative forage value, 1.52 vs 0.86 net energy for maintenance (Mcal/ $\mathbf{k g}$ ), respectively for early and late bloom. It is concluded that early bloom canola forage represents better quality because of its protein content and fiber fraction. Estimated energetic value is 1.8 times greater in early bloom compared with late bloom (1.52 vs 0.86 Mcal $\mathrm{ENm} / \mathrm{kg}$ ); and early bloom energetic value is compared with sudan grass.
\end{abstract}

KEY WORDS: Canola, Forage potential, Nutritional profile, Protein, Energy.

\footnotetext{
a Unidad Académica de Medicina Veterinaria y Zootecnia, Universidad Autónoma de Zacatecas, Zacatecas 98500. México.

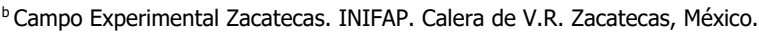

c Facultad de Ecología y Zootecnia, Universidad Autónoma de Chihuahua, Chihuahua. México.

*Autor de correspondencia: gtzbahector@hotmail.com.
} 


\section{INTRODUCCIÓN}

En el estado de Zacatecas, las siembras de frijol y maíz representan hasta el $70 \%$ del total de la superficie de temporal, de las cuales se reporta siniestrada año con año del 15 al $40 \%$, mayormente por sequía ${ }^{(1)}$. La canola representa una alternativa de siembra, es resistente a las bajas temperaturas y las sequías, requiriendo hasta $40 \%$ menos agua que el maíz ${ }^{(2)}$. El mayor uso de la canola es para la obtención de aceite y harina. No obstante, las tierras agrícolas son una oportunidad para obtener forraje adicional y satisfacer los requerimientos nutricionales del ganado. El ganado bovino en pastoreo extensivo necesita al menos $7 \%$ de proteína cruda para el mantenimiento de las poblaciones microbianas ruminales ${ }^{(3)}$ y 9 Mcal por día de energía neta de mantenimiento ${ }^{(4)}$. La determinación del valor nutricional del forraje se basa principalmente en su valoración proteica y energética. Su estimación energética es compleja, y se ha utilizado el análisis de fibras de Van Soest como un indicador para predecir en forma cuantitativa, mediante ecuaciones de predicción, la energía y el potencial de los forrajes ${ }^{(5,6)}$. Existe información limitada donde se describe el perfil nutricional, y por lo tanto, el potencial del forraje de canola bajo condiciones de temporal, lo que genera la hipótesis de que la presente oleaginosa es una alternativa forrajera para el ganado.

El objetivo del estudio fue evaluar el perfil bromatológico y nutricional del forraje de canola (Brassica napus L.) en floración temprana (5\%) y tardía $(90 \%)$ en condiciones de temporal en Zacatecas.

\section{MATERIAL Y METODOS}

Cuarenta y ocho muestras (24 por floración) se recolectaron al azar de una parcela de canola, variedad Hyola 401, que se sembró el 11 de septiembre de 2013, con una densidad de siembra de $2.5 \mathrm{~kg} / \mathrm{ha}$. La dosis de fertilización fue de 100-50, nitrógeno y fósforo, respectivamente. La mitad del nitrógeno y todo el fósforo se aplicaron al momento de la siembra, mientras que el nitrógeno restante fue en la primera escarda(7). Los días de siembra a cosecha para floración temprana fueron 52, mientras que para floración tardía 89.
Las muestras se llevaron al laboratorio de análisis bromatológicos que se encuentra en la Facultad de Medicina Veterinaria y Zootecnia, Universidad Autónoma de Zacatecas, para ser deshidratadas en un horno a $65^{\circ} \mathrm{C}$ durante $48 \mathrm{~h}$. Posteriormente, se molieron con una criba de $1 \mathrm{~mm}$. A las muestras se les determinó la cantidad de cenizas, incinerándolas a $550{ }^{\circ} \mathrm{C}$ por $6 \mathrm{~h}$, y por diferencia entre la materia seca (MS) y la ceniza se obtuvo la materia orgánica (MO). La proteína cruda (PC) se obtuvo mediante la determinación de nitrógeno total $(\mathrm{N})$ por análisis de combustión (Leco PF-428, Leco Corporation, St. Joseph, MI). Las soluciones de fibra detergente neutro (FDN) y fibra detergente ácido (FDA) se llevaron a cabo secuencialmente mediante el análisis de fibras detergentes de Van Soest ${ }^{(8)}$, utilizando el analizador de fibras Ankom 200 (Ankom Technology). La hemicelulosa (HEM) se obtuvo mediante la diferencia entre FDN y FDA.

\section{Perfil nutricional y potencial forrajero}

Los siguientes valores nutricionales se calcularon utilizando los resultados bromatológicos. La energía neta para mantenimiento (ENm) y la energía neta para lactancia (ENI) se obtuvieron utilizando la ecuación 1.037 - $0.0124 *$ FAD y multiplicando el resultado por 2.202 para ajustarlo a kilos $^{(4)}$. El total de nutrientes digestibles se obtuvo mediante la ecuación $8+86 * \mathrm{ENI}^{(4)}$. El consumo de materia seca en base al porcentaje de peso corporal del animal (DMI, \%BW) se calculó como 120 / (\%FDN). La digestibilidad de la materia seca (DMS, $\%$ DM) se determinó como 88.9 - $0.779 *$ (FDA), y el valor relativo del forraje (RFV) se calculó como DMI * DMS / 1.29(5).

\section{Análisis estadístico}

El análisis se realizó con el procedimiento PROC MIXED del paquete estadístico $\operatorname{SAS}^{(9)}$ y las medias se compararon con prueba de Tukey al $5 \%$ de probabilidad. Para determinar las posibles relaciones entre pares de variables, se estimó el coeficiente de correlación de Pearson mediante PROC CORR del paquete estadístico SAS, y se eligieron aquéllas que presentaron probabilidad mayor al $95 \%$. 


\section{RESULTADOS Y DISCUSIÓN}

\section{Evaluación bromatológica}

En el Cuadro 1 se presentan los valores nutricionales del forraje de canola en floración temprana y tardía. Se observaron diferencias en todas las variables $(P<0.01)$. Los niveles de proteína de la canola, independiente de la etapa de floración, se consideran por arriba de los requerimientos de mantenimiento del bovino, los cuales se calculan entre el 7 y $8 \%{ }^{(3)}$. En las explotaciones de ganado existe una gran variabilidad en la calidad nutricional de los forrajes, por lo que en algunos casos, sus características nutricionales están por debajo de las recomendaciones nutricionales. El aporte total de proteína de esta oleaginosa depende del nivel de inclusión de la dieta. La mayoría de los cereales contienen del 6 al $10 \%$ de $\mathrm{PC}^{(6)}$, por lo que inclusive la canola en floración tardía representa valores mayores. La disminución en el contenido de proteína cruda es el resultado del desarrollo de la planta, que al madurar sus órganos estructurales tales como tallos y peciolos, fomenta la disminución de contenidos celulares, lo que promueve la dilución del $\mathrm{N}$ de la planta ${ }^{(10,11)}$. Los requerimientos de proteína para un bovino de tamaño mediano de $182 \mathrm{~kg}$ en crecimiento son de 9 a $16 \%{ }^{(12)}$, por lo que la canola representa en cualquier estado de floración (dependiendo la tasa de ganancia del animal y el nivel de consumo de forraje) una buena fuente de proteína para el ganado.

Los valores de FDN en floración tardía pueden resultar en efectos negativos en el consumo de materia seca por un efecto del llenado del rumenretículo(11). Cuando el forraje llega a su estado de madurez, el contenido de nutrientes digestibles disminuyen, aumentando los componentes de la pared celular (celulosa, hemicelulosa y lignina) ${ }^{(12,13)}$. Estos valores nutricionales se asocian con características morfo fisiológicas de la planta, que varían con la especie, variedad, clima, suelo, entre otros, pero fundamentalmente con el estado de madurez $^{(14,15)}$. La lignificación de las plantas es el principal factor que afecta los demás componentes nutricionales. Se observa una relación, entre mayor madurez de la planta, se incrementa la cantidad de lignina. Un indicador de la lignina presente en las plantas es la cantidad de FDA, los niveles en floración tardía se presentaron en $52 \%$. El contenido de FDA es una cuantificación de la fracción indigerible. En la canola la FDA varía con el tiempo de cosecha, observándose valores menores en la etapa inicial y valores mayores en la etapa

Cuadro 1. Valor nutritivo del forraje de canola (Brassica napus L.) en floración temprana y tardía bajo condiciones de temporal

\begin{tabular}{lcccc}
\hline Variable & Floración temprana & Floración tardía & $P$ & EE \\
\hline Cenizas, \% BS & 17.83 & 9.86 & $<0.001$ & 0.54 \\
MO, \% BS & 82.17 & 90.14 & $<0.001$ & 0.54 \\
PC, \% BS & 26.32 & 12.36 & $<0.001$ & 0.50 \\
FND, \% BS & 47.09 & 60.42 & $<0.001$ & 1.04 \\
FAD, \% BS & 27.83 & 52.06 & $<0.001$ & 1.02 \\
HEM, \% BS & 19.27 & 8.57 & $<0.001$ & 1.02 \\
ENm, Mcal/kg BS & 1.52 & 0.86 & $<0.001$ & 0.01 \\
NDT, \% BS & 67.51 & 41.67 & $<0.001$ & 1.08 \\
DMI, \% PV & 2.56 & 1.99 & $<0.001$ & 0.04 \\
DMS, \% & 67.22 & 48.35 & $<0.001$ & 0.79 \\
VRF & 133.37 & 71.59 & $<0.001$ & 4.28 \\
\hline
\end{tabular}

$\mathrm{BS}=$ base seca; $\mathrm{MO}=$ materia orgánica; $\mathrm{PC}=$ proteína cruda; $\mathrm{FND}=$ fibra neutro detergente; $\mathrm{FAD}=$ fibra ácido detergente $\mathrm{HEM}=$ hemicelulosa; $E N m=$ energía neta de mantenimiento; ENg= energía neta de ganancia NDT= nutrientes digestibles totales DMI= consumo de materia seca DMS= digestibilidad de la materia seca RFV= valor relativo del forraje. 
final. Este comportamiento es similar a otras plantas forrajeras, en las cuales se ha reportado que conforme la planta madura, su contenido de FDA aumenta, y la ingestión y digestibilidad de la materia seca se reducen ${ }^{(16)}$. Por otro lado, los niveles de FDA presentes en la canola en floración temprana se consideran importantes para mantener los procesos fisiológicos del rumen. La FDA es el mejor indicador de los requerimientos de fibra para una fermentación saludable en el rumen. El $\mathrm{NRC}^{(12)}$ indica que las raciones del ganado lechero deben contener 19 a $27 \%$ de FDA. Si el suplemento es menor, el contenido de grasa en la leche puede disminuir. La cantidad de FDA se ha correlacionado con la fibra efectiva, la cual mantiene en equilibrio la microbiota ruminal, mediante la estimulación de la rumia, secreción de saliva, reciclamiento del nitrógeno, y por consiguiente una estabilidad en el $\mathrm{pH}$ ruminal, lo cual genera mejores condiciones para la fermentación ${ }^{(17)}$. En el presente estudio, sólo la canola en floración temprana aporta niveles adecuados de FDA, por lo que las altas cantidades en la floración tardía disminuyen la digestibilidad de esta oleaginosa.

\section{Perfil nutricional}

Los nutrientes digestibles obtenidos en la floración temprana son $26 \%$ mayores que en la floración tardía. La digestibilidad de la canola en floración temprana (67\%) se pudiera ver reflejada en tasas moderadas de aumento de peso corporal y de producción de leche ${ }^{(18)}$. Los nutrientes digestibles totales (NDT) y la digestibilidad de la materia seca (DMS), han sido usados como indicadores para estimar el comportamiento productivo del ganado alimentado con forrajes. Los valores de FDA $(P<0.01)$ comienzan bajos en la canola en floración temprana (28\%), para después aumentar en floración tardía (52\%), disminuyendo drásticamente la DMS (67 vs $48 \%$ ); esta disminución está asociada con el aumento de la edad de las plantas y la lignificación de las paredes celulares, por lo tanto, son más fibrosas y menos digeribles ${ }^{(19)}$, lo que se ve reflejado también en una disminución drástica en la energía disponible para el ganado conforme aumenta la cantidad de fibras. La ENm en floración temprana es similar al pasto sudán, el cual contiene valores de $1.47 \mathrm{Mcal}$ ENm/kg. Sin embargo, la ENm en floración tardía disminuye en $43 \%$ comparado con la floración temprana, con valores similares a la grama común (Cynodon dactylon), de $0.93 \mathrm{Mcal}^{(6)}$. Los resultados indican un perfil energético para satisfacer los requerimientos de mantenimiento y moderadas tasas de ganancia( ${ }^{(6)}$. Algunos autores $^{(20,21,22)}$ utilizan las variables NDT y valor relativo del forraje (VRF) como indicadores para evaluar la calidad de henos de leguminosas o gramíneas, o para la asignación de forraje a grupos de animales de acuerdo a los requerimientos nutricionales. Para los requerimientos del ganado productor de carne los valores obtenidos de floración temprana, cubrirían los requerimientos de becerros de 136 a $318 \mathrm{~kg}$ con una ganancia diaria de $900 \mathrm{~g}^{(6)}$. Por lo tanto, la canola en floración temprana tiene mejor calidad nutritiva y mayor productividad animal, debido a los niveles mayores de PC, bajos contenidos de fibras, y mayores aportaciones en energía.

Los coeficientes de correlación entre las variables se presentan en el Cuadro 2 . El VRF se correlacionó positivamente con la PC, NDT, y negativamente con FND y FAD. Se aprecia que a mayor cantidad de PC el VRF es mayor, al igual que a mayor cantidad de NDT. A mayor cantidad de fibras es menor el VRF. Los NDT se correlacionaron positivamente a mayor grado con ENI, ENm y ENg. Las fibras FND y FAD se correlacionaron negativamente con ENI, ENm, y ENg. En lo anterior se observa que a mayor cantidad de fibras menor energía(23). La FAD es la parte menos digestible de la planta, por ende, la mayor parte de ésta aparece en el excremento.

\section{CONCLUSIONES E IMPLICACIONES}

La canola en floración temprana presenta mayores niveles de proteína cruda comparada con floración tardía. Sin embargo, los niveles elevados de FDN y FDA en floración tardía disminuyen las predicciones de digestibilidad y la cantidad de energía disponible. Estudios posteriores deberán evaluar el efecto de la lignificación de la canola en floración tardía sobre la digestibilidad de la proteína cruda, y la inclusión de esta oleaginosa a diferentes niveles de la dieta en el ganado. 
Cuadro 2. Correlación de Pearson entre variables de perfil bromatológico y nutricional

\begin{tabular}{|c|c|c|c|c|c|c|c|c|}
\hline & PC & FND & FAD & NDT & ENI & ENm & ENg & VRF \\
\hline$P C$ & 1 & . & - & - & - & - & - & $\begin{array}{c}0.91175^{\star} \\
<.0001\end{array}$ \\
\hline FDN & & 1 & - & - & $\begin{array}{c}-0.91989^{*} \\
<.0001\end{array}$ & $\begin{array}{c}-0.91989^{*} \\
<.0001\end{array}$ & $\begin{array}{c}-0.93961^{*} \\
<.0001\end{array}$ & $\begin{array}{c}-0.88666^{*} \\
<.0001\end{array}$ \\
\hline FDA & & - & 1 & - & $\begin{array}{l}-0.998^{\star} \\
<.0001\end{array}$ & $\begin{array}{l}-0.998^{*} \\
<.0001\end{array}$ & $\begin{array}{c}-0.98307^{*} \\
<.0001\end{array}$ & $\begin{array}{c}-0.97713^{\star} \\
<.0001\end{array}$ \\
\hline NDT & & - & - & 1 & $\begin{array}{l}0.998^{*} \\
<.0001\end{array}$ & $\begin{array}{l}0.9987^{\star} \\
<.0001\end{array}$ & $\begin{array}{c}0.98307^{*} \\
<.0001\end{array}$ & $\begin{array}{c}0.97713^{*} \\
<.0001\end{array}$ \\
\hline
\end{tabular}

$\mathrm{PC}=$ proteína cruda $\mathrm{FND}=$ fibra neutro detergente $\mathrm{FAD}=$ fibra ácido detergente; $\mathrm{ENm}=$ energía neta de mantenimiento; $\mathrm{ENg}=$ energía neta de ganancia; $\mathrm{NDT}=$ nutrientes digestibles totales VRF= valor relativo del forraje.

\section{AGRADECIMIENTOS}

Este trabajo fue subsidiado parcialmente por el proyecto PROMEP fortalecimiento a los cuerpos académicos con registro UAZ-2012-36067.

\section{LITERATURA CITADA}

1 Luna FM, Hernández MJ, Luna EMG, de Santiago Z, Humberto L, García HS. Los cultivos de frijol y maíz de grano bajo condiciones de secano en Zacatecas, México de 1980 a 2008. Rev Mex Cienc Agríc 2012;3(2):327-338.

2. Oleaginosas. Los agricultores que siembran oleaginosas ahorran agua. Oleaginosas en cadena. 2009; No. 24. www.oleaginosas.org/ cargas/boletin_24.pdf, Consultada 12 Sep, 2016.

3. Van Soest PJ. Nutritional ecology of the ruminant. 2nd ed. Ithaca, NY; Comstock, Cornell University Press; 1994.

4. Belyea RL, Steevens B, Garner G, Whittier JC, Sewell H. Using NDF and ADF to balance diets. 1993. http://extension.missouri.edu/p/ G3161. Accessed Sep 13, 2015.

5. Moore JE, Undersander J. Relative forage quality: An alternative to relative feed value and quality index. Proc Ann Florida Rum Nut Symp. 2002;32:16-29.

6. NRC. National Research Council. The nutrient of requirements of beef cattle. 7th ed. Washington, DC, USA: National Academy Press; 2000.

7. Zandate HR, Medina GG. Guía para la producción de canola en Zacatecas. Campo Experimental Zacatecas. CIRNOC-INIFAP. Folleto para productores No. 36. 2010.

8. Goering HK, Van Soest PJ. Forage fiber analyses. Apparatus, reagents, procedures, and some applications. Agric 1970. Handbook 379.
9. SAS. User Guide. Statiscal analysis system. Inc. Cary, NC. Version 9.1. 2002.

10. Taize L, Zerge E. Fisiología vegetal. $3^{a}$ ed. Porto Alegre, Brasil: Artemet; 2004.

11. Müller L, Manfron PA, Santos OS, Medeiros SLP, Neto NN, Morselli TBGA, et al. Efecto de soluções nutitives na produ- ção e qualidade nutricional da forragem hidropônica de trigo (Triticum aestivum $\mathrm{L}$ ). Zootec Trop 2006;24:137-152.

12. NRC. National Research Council. The nutrient requirements of Dairy cattle. 6th ed. Washington, DC, USA: National Academy Press; 1989.

13. Bosch MW, Tamminga S, Post G, Leffering CP, Muylaert JM. Influence of stage of maturity of grass silages on digestion processes in dairy cows. 1. Composition, nylon bag degradation rates, fermentation characteristics, digestibility and intake. Livest Prod Sci 1992;32(3):245-264.

14. Rotz CA, Muck RE. Changes in forage quality during harvest and storage. In: Forage quality, evaluation, and utilization. Madison, WI; Am Soc Agron 1994:828-868.

15. Van Soest PJ. Environment and forage quality. Cornell Nutrition Conference for Feed Manufacturers (USA). 1996.

16. Van Soest PJ, Mertens DR, Deinum B. Preharvest factors influencing quality of conserved forage. J Anim Sci 1978;47:712-720.

17. Nocek JE. Bovine acidosis: Implications on laminitis. J Dairy Sci 1997;80(5):1005-1028.

18. Marston TT, Blasi DA, Brazle FK, Kuhl GL. Beef cow nutrition guide. Kansas State University, Agr Exp Sta Coop Ext Service. 1998.

19. Urriola DM. Efecto de la edad de rebrote sobre la composición química y digestibilidad in vitro de cinco procedencias de Gliricidia sepium (Jacq.) y su aceptabilidad por cabras adultas. [tesis maestría]. CATIE. Turrialba, Costa Rica; 1994. 
20. Carr PM, Horsley RD, Poland WW. Barley, oat and cereal pea mixtures as dryland forages in the northern plains. Agro J 2004;(96):677-684.

21. Strydhorts SM, King JR, Lopetinsky KJ, Harker KN. Forage potential of intercropping barley with faba bean, lupin, or feal pea. Agron J 2008;(100):182-190.
22. Sánchez GRA, Gutiérrez BH, Serna PA, Gutiérrez LR, Espinoza CA. Producción y calidad de forraje de variedades de avena en condiciones de temporal en Zacatecas. Rev Mex Cienc Pecu 2014;5(2):131-142.

23. Hutjens M. Guía de alimentación. $2^{\mathrm{a}}$ ed. Wisconsin, USA: Hoard's Dairyman en Español 2003. 